\title{
HIGH RESOLUTION IMAGING FORTY YEARS AGO
}

\author{
R. C. JENNISON \\ Emeritus Professor, University of Kent at Canterbury, U. K.
}

This conference is concerned with the very high resolution imaging of cosmic sources in many parts of the electromagnetic spectrum. Various techniques are now available and the equipment is often automated and highly sophisticated but the term 'very high angular resolution' is comparative. Many of the problems existed over forty years ago when the best resolving power was about half a degree and the two major radio 'stars' appeared to be point sources. Very high resolution imaging in those days was the struggle to reach one minute of arc and Hanbury Brown had set his sights on considerably better than one second of arc with the concept of the intensity interferometer. The dream was to achieve a resolving power comparable to that of optical telescopes.

In 1952, both Mills, in Australia, and Jennison and Das Gupta in England, were independently using radio linked systems and working with baselines well in excess of 2000 wavelengths (Mills, 1952; R. Hanbury Brown, Jennison and Das Gupta, 1952). Graham Smith, in Cambridge, applied the superb engineering of that establishment, together with accurate measurement techniques, to detect a decrease in the visibility function of Cassiopeia at much shorter baselines (Smith, 1952). Mills resolved Cygnus but did not detect the structure. Jennison and Das Gupta ${ }^{1}$ played a slightly different game and obtained the first high resolution images of both Cassiopeia and Cygnus by measuring the visibility on three baselines crossing at 120 degrees. The site of the first outstation was within the confines of Jodrell Bank and it was fully expected that the correlation would be $100 \%$ but, to our surprise, both sources exhibited slightly lower values, i.e., both sources appeared to be partially resolved at this very small spacing! The baseline was extended along roughly the same azimuth to the paddock adjoining Bernard Lovell's house, a distance of 3.99 $\mathrm{km}$. Cassiopeia was completely resolved whilst the correlation of Cygnus fell to $79 \%$. When we 'boxed in' the sources with measurements on the other two baselines and plotted the angular width of the equivalent strips, Cassiopeia appeared approximately circular whilst Cygnus was smaller, narrower and anomalous (Smith, 1952).

The anomaly in the Cygnus measurements, reproduced approximately in figure 1, was important, for we had considerable faith in the accuracy of our measurements

\footnotetext{
${ }^{1}$ Hanbury, who was entirely responsible for the initial concept, was, unfortunately, in Australia and the United States at this critical time, so Das Gupta and I did what we thought was best and made measurements on three axes. The angular diameters of Cygnus and Cassiopeia could have been measured at least six months earlier with the intensity interferometer but Hanbury had, very reasonably, decided that we had to prove that the system worked by measuring the angular diameter of the quiet sun. Despite the fact that it was near sun spot minimum, the sun still had numerous small spots which interfered severely with the diameter measurements and delayed the major experiment. A short account of this and details of the first intensity interferometer appear in Jennison and Latham (1959). It was, of course, expected that Cygnus and Cassiopeia might subtend only a fraction of a second of arc, so that it appeared unlikely that the answer lay in the range of the Michelson interferometers of that era. In Hanbury's later words: "We used a steamroller to crack a nut!"
} 


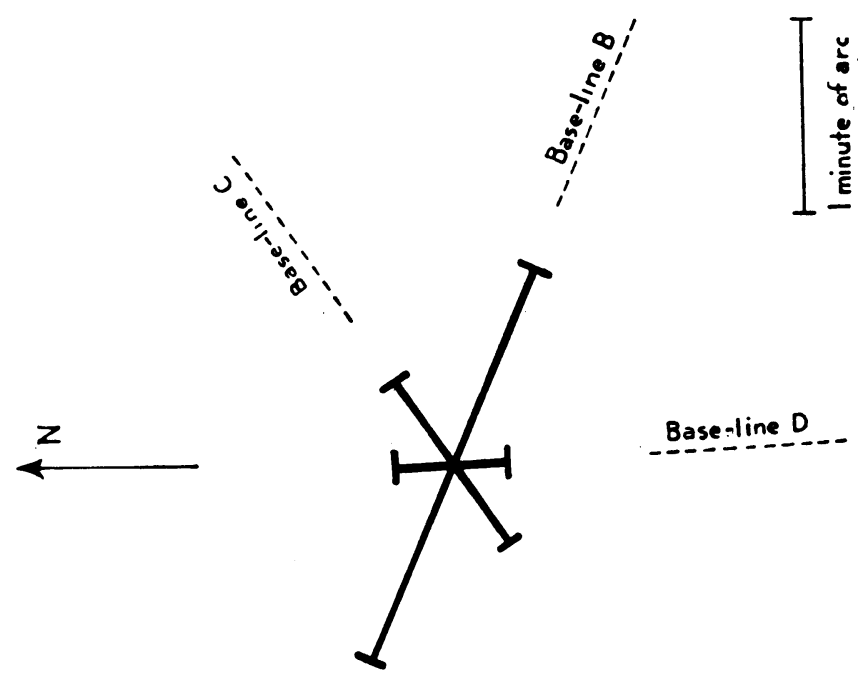

Fig. 1. Equivalent angular width of the source in Cygnus observed from different base-lines. The value shown is the width of an equivalent rectangular strip of constant surface intensity (reprinted from R. H. Brown, Jennison and Das Gupta, 1952).

and I could not reconcile the measurements in three directions with the projection of a single object of any chosen shape. To quote from our 1952 paper ( $R$. Hanbury Brown, Jennison and Das Gupta, 1952): "A preliminary analysis indicates that the results are incompatible with a source of simple elliptical shape and constant surface density, and that a more complicated model must be used" It was then that I realised that the readings fell perfectly into place if Cygnus was a double source. This little part of the story is not well known, nor indeed well understood by many people, who assume that the double structure was deduced from extensive measurements of the visibility function on or near the major axis. It is an amusing moral in the light of present day sophisticated analysis and techniques.

Having already decided that Cygnus was double, we set about determining the major axis with some precision, and then measured the correlation function along that axis, from which we confirmed the double structure, with readings which were accurate to within a few seconds of arc, and only then did we publish the result (Jennison and Das Gupta, 1953).

One might say that this crude result, showing the two dimensional picture of a double source reproduced in Figure 2, was the first real high resolution image to be derived from stellar interferometry. It was obtained with an economy of technique, 


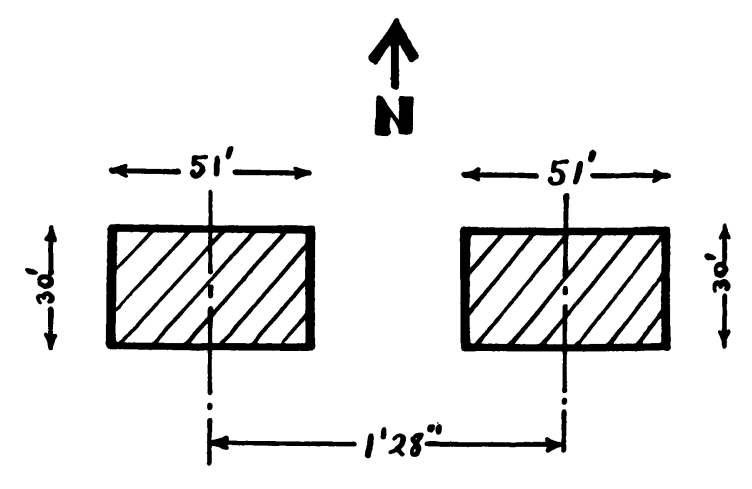

Fig. 2. Approximate intensity distriubtion of the extra-terrestrial radio source in Cygnus (reprinted from Jennison and Das Gupta, 1953).

by choosing the spacings along the major axis in the light of the information gleaned from figure 1 and the subsequent axial measurements. In particular it was important to determine the accurate values around the first minimum to determine if there was any observable filling from a weaker central source. The mobile antenna, contrary to Hanbury's recollection (Hanbury Brown, 1991), was identical to that at the base station $(120 \mathrm{ft} \times 41 \mathrm{ft})$ and it could not be moved to precise small increments of the baseline in the unfavourable terrain of the undulating and populated Cheshire countryside. I therefore devised a simple technique to obtain clusters of points at slightly different baselines from each site. This depended on the fact that the intrinsic source structure was unlikely to change rapidly with wavelength. We than operated the system using three closely adjacent frequencies from which we obtained the gradient of the correlation function.

Nevertheless, the Cygnus structure was received with some reserve by the scientific community. I do not think that Ron Bracewell ever understood how I was able to derive the original information from figure 1 and there were severe problems in performing further useful measurements with the intensity interferometer. It was extremely insensitive (the output varied as the square of the visibility function) and it was incapable of measuring phase. Most of the radio astronomers at this time were interested in surveys at high sensitivity and had designed carefully phased arrays on relatively short baselines. No one else was interested in high resolution structures and there was a problem. How could one measure the unambiguous structure of the smaller radio sources when the antennae had to be moved to various sites on a very undulating terrain and there were unknown phases associated with the ionosphere, the receiving equipment and the radio links? This problem was very daunting. One had to extract the phase of the transform of the source distribution from a whole mixture of other unknown phases. The solution came to me in the autumn of 1952 (not 1951, as sometimes quoted) when I realised that one was really dealing with 
a set of systematic errors and that it should be possible to eliminate systematic errors by redesigning the basic method of measurement. That was the birth of the technique now known as closure phase or phase closure, according to which way round you look at it. In 1953/4 I built the basis of the prototype equipment, in which I incorporated variable speed phase rotators in two of the channels (Jennison 1957,1958$)$. The system used the existing intensity interferometer antenna at Jodrell Bank and two smaller portable antennae for the outstations. I built a new pair of radio links to transmit the signals from the two outstations back to base. Unlike the radio links later used by Henry Palmer at Jodrell Bank, these radio links were entirely home made and operated, illicitly, on one of the television channels dedicated to a distant part of the U.K. Vincent Latham joined me as a research student to help in the later stages of construction and observations and together we were able to confirm and refine the image of Cygnus at $127 \mathrm{MHz}$ (Jennison, 1957) and to discover an asymmetric spur protruding about four minutes of arc beyond the limb of Cassiopeia A (Jennison and Latham, 1959). I communicated this finding to Minkowski, who photographed Cassiopeia again with the 200 inch Palomar telescope and confirmed the observation. This flare has now virtually disappeared but I have reports from the United States that it was still detectable at low frequencies in the mid nineteen sixties. The remnant of Cassiopeia still shows the scar of the site of the flare but even that is changing, after all Cassiopeia is now about $12 \%$ larger than when I first measured it in 1952 !

The observation of the Cassiopeia flare probably marks the first observation of asymmetry in the high angular resolution of the radio sources. Shortly after this, Lovell wanted all activities to incorporate the $250 \mathrm{ft}$ telescope, ${ }^{2}$ which was nearing completion. I gave up further work on closure phase and high resolution source structures and reluctantly worked for a while on the big dish, prior to moving to other interests. I did, however, design and construct an elementary version of an optical phase closure interferometer, which I set up in the final year teaching laboratory of the Physics Department at Manchester University, where it was used by some of the students. I published an account of this instrument in 1961 (Jennison, 1961).

\section{References}

Mills, B.Y.: 1952, Nature 170, 1063.

Smith, F. G.: 1952, Nature 170, 1063.

Brown, R. Hanbury, Jennison R.C. and Das Gupta M.K.: 1952, Nature 170, 1061.

Jennison, R.C. and Das Gupta, M.K.: 1953, Nature 172, 996.

Jennison, R. C.: 1953, in Symposium on Radio A stronomy at Jodrell Bank, R. H. Brown, ed., The Observatory 73, 190-191.

Jennison, R.C. and Das Gupta, M.K.: 1956, Phil Mag 1, 55.

Jennison, R.C. and Das Gupta, M.K.: 1956, Phil Mag 1, 65.

${ }^{2}$ Lovell, for fairly good reasons, was hypersensitive about the publication of any work at Jodrell Bank which was not directly connected with the embryonic $250 \mathrm{ft}$ dish, which was then in the stages of final design, and there were severe funding problems. If one submitted a draft paper to him for approval it usually had to go through at least two or three revisions before he found it acceptable. This treatment was not conducive to the writing up of results and, consequently, a number of the papers listed here were some years in the pipeline! 
Hanbury Brown, R.: 1991, Boffin, Adam Hilger, Bristol, 107.

Jennison, R.C.: 1958, Mon. Not. R. astr. Soc. 118, 174.

Jennison, R. C.: 1957, in IAU Symposium No. 4 on Radio Astronomy, H. C. van der Hulst, ed., Cambridge University Press, 159.

Jennison, R.C. and Latham, V.: 1959, Mon. Not. R. astr. Soc. 119, 174.

Jennison, R.C.: 1961, Proc. Phys. Soc. 78, 596. 